\title{
Comment on: Medical student mistreatment by patients in the clinical environment - a student perspective
}

Singapore Med J 2019; 60(11): 606 https://doi.org/10.11622/smedj.2019150

Dear Sir,

We read with great interest the article by Zhu et al regarding medical student mistreatment. ${ }^{(1)}$ As final-year medical students at King's College London, we appreciate your attention to the subject, which affects students universally. We write to offer anecdotal evidence on low-level reporting.

The authors indicate that students may not recognise the severity of incidents. We agree that the lack of specificity around mistreatment makes it hard to acknowledge when it occurs. Where mistreatment is obvious, we are taught to apologise, de-escalate and exit, (2) with emphasis on patient safety rather than student well-being. This behaviour is reinforced by a hospital culture of tolerance, where doctors brush aside mistreatment as 'part of the job' or 'no big deal'.(3) As students seek to emulate their seniors, watching doctors minimise mistreatment diminishes the importance of reporting it. The authors also suggest that students may lack awareness of the reporting process but, considering the above, even those who are aware may be reluctant to give feedback to staff. ${ }^{(4)}$ We choose instead to discuss these encounters with our peers, who can empathise with our experiences.

To promote positive reporting and discourage stress internalisation, we suggest implementing discussion groups for peers and trusted faculty, or 'Schwartz Rounds'. ${ }^{(5)}$ Online forums or e-mentoring are alternatives, to maintain anonymity. These outlets are offered at King's and we feel empowered to seek help following difficult encounters. Simultaneously, offering students seminars on identifying mistreatment and emphasising the importance of reporting would be a positive step in combatting the tolerance culture.

Yours sincerely,

Afra Цiwa ${ }^{1}$, Armin $\underline{\text { Fardanesh }}^{1}$, Stavroula Stavropoulou-Tatla ${ }^{1}$

${ }^{1}$ King's College London School of Medical Education, Guy’s Campus, Great Maze Pond, London, United Kingdom. afra.jiwa@kcl.ac.uk

\section{References}

1. Zhu G, Tan TK. Medical student mistreatment by patients in the clinical environment: prevalence and management. Singapore Med J 2019; 60:353-8.

2. Vishwanathan K. Dealing with angry and aggressive patients. BMJ 2006; 333:s64.

3. British Medical Association. Bullying and harassment: how to address it and create a supportive and inclusive culture. Available at: https://www.bma.org.uk/-/media/ files/pdfs/collective\%20voice/policy\%20research/education\%20and\%20training/bma-bullying-and-harassment-policy-report.pdf?la=en. Accessed October 17, 2019.

4. Chung MP, Thang CK, Vermillion M, Fried JM, Uijtdehaage S. Exploring medical students' barriers to reporting mistreatment during clerkships: a qualitative study. Med Educ Online 2018; 23:1478170.

5. Angoff NR, Duncan L, Roxas N, Hansen H. Power day: addressing the use and abuse of power in medical training. J Bioeth Inq 2016; 13:203-13. 